\title{
La correlación del clítico SE y las preposiciones a/para en verbos de movimiento intransitivos en el español del siglo XIII
}

\author{
The correlation between the clitic SE and the prepositions a/para \\ in intransitive motion verbs of the 13th century Spanish
}

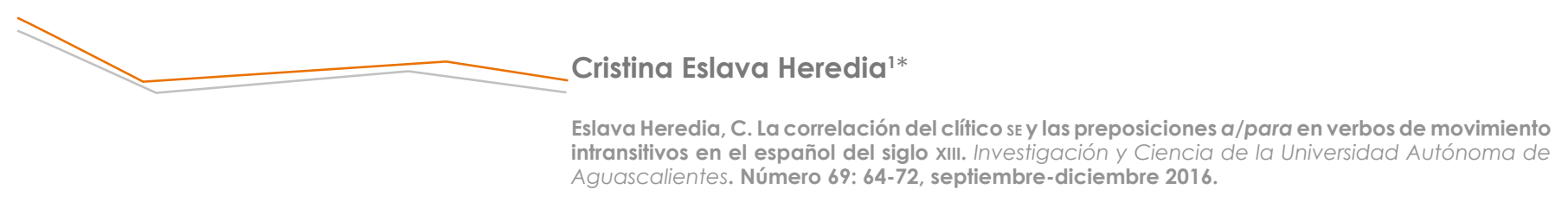

RESUMEN

El artículo ${ }^{2}$ trata de la alternancia de las preposiciones a/para en la formación de la Meta en construcciones con verbos de movimiento intransitivos del español del siglo XIII. Se hace énfasis en la correlación entre la presencia de un clítico pronominal se y la elección de una preposición directiva como preconfiguración de la Meta: como argumento al elegir a o como no-argumento al elegir para. La elección de para constituiría un paso intermedio de la función del clítico como "habilitador de categoría vacía» que se manifiesta en etapas posteriores (p. e. Juan fue al parque/*Juan fue/Juan se fue para el parque/ Juan se fue).

\section{ABSTRACT}

This work presents the alternation of the prepositions a/para when these perform the goal locative complement in structures constructed with intransitive motion verbs in 13th century Spanish. The emphasis

Palabras clave: verbos de movimiento, clítico, preposiciones a/para , siglo XIII.

Keywords: motion verbs, pronoum, prepositions a/ para, 13th century.

Recibido: 26 de octubre de 2015, aceptado: 16 de junio de 2016

1 Departamento de Letras, Centro de las Artes y la Cultura, Universidad Autónoma de Aguascalientes.

* Autor para correspondencia: hceslava@correo.vaa.mx

2 La autora agradece los comentarios y correcciones de la Dra. Blanca Elena Sanz Martin y la asistencia de Mario Antonio Frausto Grande en la elaboración de este trabajo. El contenido de este artículo es, por supuesto, reponsabilidad totalmente de la autora.

3 Agradezco a los dictaminadores anónimos la sugerencia de usar este término, pues ayuda a no confundir el uso del clítico con un clausurador de argumento per se. is placed on the correlation between the presence of the pronominal clitic se and the choice of a directive preposition as a preconfiguration of the goal: as argument with the preposition a or not argument with the preposition para. The choice of the preposition para is an intermediate step towards the clitic as an "empty category habilitator", present in subsequent stages of the language (e. g. Juan fue al parque./*Juan fue/Juan se fue para el parque/Juan se fue).

INTRODUCCIÓN

El estudio de construcciones con verbos de movimiento ha sido de gran interés desde diferentes perpectivas sincrónicas y diacrónicas. A partir de estos estudios se sabe que el significado cognitivo de movimiento supone al menos tres fases: el origen o Fuente, la ruta o el movimiento mismo y el destino o Meta (veáse los trabajos de Talmy, 1975, 1985; Crego García, 2000; Ibáñez Cerda, 2005; García Miguel, 2006; entre otros). Según lbáñez Cerda (2002, 2005), algunos verbos son capaces de integrar en su construcción la Fuente y la Meta, en tanto que comparten un mismo marco conceptual (Fillmore, 1982); por ejemplo, en español actual las formas: ir, venir, salir y regresar formalizan ambos locativos, tal como se ejemplifica en las oraciones de (1-4):

(1) Juan fue de la escuela a la casa.

(2) Juan vino del trabajo a la Universidad.

(3) Juan salió del restaurante al mirador.

(4) Juan regresó del gimnasio a su casa.

Ibáñez Cerda (2002: 170) reconoce que, ciertamente, es posible construir oraciones que expresen la 
Fuente y la Meta, en una misma oración, como los casos anteriores de (1-4) y el ejemplo de (5a). Sin embargo, lo que señala el autor es que en el uso real, casi siempre se prescinde de la Fuente más que de la Meta. El ejemplo de (5b) es gramatical aunque no aparezca la Fuente formalizada; mientras que en (5c), la carencia de la Meta hace la oración agramatical.

(5) a. Luis fue de Puebla a Tehuacán

b. Luis fue a Tehuacán

c. *Luis fue de Puebla (Ibáñez Cerda, 2002: 170)

El autor observa que el uso del uclítico posibilita la aparición de la Fuente cubriendo el hueco que el verbo no pronominal evidenciaı) (Ibáñez Cerda, 2002: 170), tal como en el ejemplo de (6) .

(6) Luis se fue de Puebla

Entonces, el autor afirma que «mientras que ir pone especial énfasis en la Meta, irse pone en perspectiva a la Fuente») (Ibáñez Cerda, 2002: 170); es decir, propone que "la función del clítico es la de perfilar la Fuente acosta de la Meta" (Ibáñez Cerda: 171).

Una segunda propuesta desarrollada por Bogard Sierra (2006: 768) propone que el clítico en ciertos verbos de movimiento, como ir, tiene la función de marca de aspectual y ofrece el par de ejemplos siguientes:

(7) a) El señor fue a vivir a la playa

b) El señor se fue a vivir a la playa

Para el autor, la diferencia entre estas oraciones consiste en que la primera es concomitante con una lectura durativa, mientras que la segunda favorece una lectura perfectiva. La interpretación dependerá del contexto donde esté insertada la oración y ofrece los siguientes ejemplos para explicar el punto.

(8) a. El señor fue a vivir a la playa cada vez que necesitó mejorar su salud

b. ¿ै* El señor se fue a vivir a la playa cada vez que necesitó mejorar su salud

(9) a. El señor se fue a vivir a la playa cuando su salud no resistió más la altura de la capital

b. *El señor fue a vivir a la playa cuando su salud no resistió más la altura de la capital
El autor explica que en el caso de (8) el contexto agrega un sentido de duratividad, de iteratividad; mientras que en el caso de (9) hay un sentido de completud o término. En (8) la forma no marcada es aquella que no incluye el clítico en la estructura (8a), pues se asocia con una lectura iterativa, dada por la interpretación oracional del proceso que se repite, en tanto evento completo, una vez tras otra, dentro de una escala determinada de tiempo (cf. Ibáñez Cerda, 2002); mientras que la inclusión del clítico en (8b) derivará en una estructura marcada o agramatical. En (9), la construcción con clítico (9a) es la no marcada, pues, se asocia con un sentido de completud; mientras que (9b) resulta bastante marcada o agramatical, a decir del autor.

Por último, Maldonado (1993, en Ibáñez, 2002: 179-180) propone otra alternativa sobre la función del clítico en verbos de movimiento intransitivos y señala que en el par caer/caerse, el clítico funciona como un marcador pragmático de "contraexpectativa", y lo ejemplifica de la siguiente manera:

(10) a) El tiro de Jordan cayó de la canasta con toda limpieza

b) *El tiro de Jordan se cayó de la canasta con toda limpieza

En el caso de (10a), la oración es correcta en función de que la caída de la pelota es totalmente esperada, según la ley de la gravedad y, por tanto, carece del clítico; por el contrario el ejemplo (10b) es agramatical, debido a que el clítico añade un sentido de evento inesperado, el cual se ilustra mejor en el siguiente ejemplo de (11).

\section{(11) a) El florero se cayó de la mesa}

En este caso, el clítico cumple la función de "marcador de contraexpectativa", dado que expresa la violación de la permanencia esperada del florero sobre la mesa.

Para etapas tempranas del español como el siglo XIII, no obstante, la presencia del clítico no parece desempeñar la función de cambio de perspectiva de la Fuente en detrimento de la Meta como señala lbáñez Cerda (2002). Tampoco se encuentran contextos donde el clítico suponga un sentido de contraexpectativa que esté, a su vez, ligado con la presencia de la Fuente o la Meta, como señala Maldonado (1993). Como se verá más adelante, los datos del siglo XIII muestran que, aunque el clitico está presente, la 
Meta persiste en la oración; pero sí hay modificaciones en la construcción de la misma al introducir el referente locativo con la preposición para, en lugar de la preposición prototípica a.

A partir del análisis de un corpus cerrado de textos representativos del siglo XIII, se ha observado que existe una marcada alternancia entre las preposiciones a/para en construcciones con verbos de movimiento intransitivos; mientras que otras preposiciones directivas como hacia y contra manifiestan muy baja frecuencia ${ }^{4}$.

La elección de a y para como marcadores de la relación espacial Meta parece estar altamente relacionada con el clítico pronominal se en todas sus formas paradigmáticas. Como se verá a continuación, la preposición a puede ocurrir tanto con el clítico se, como sin él; mientras que la preposición para depende de la presencia del mismo. Se ponen a consideración del lector las siguientes estructuras de (12a y b).

(12) a. Yermo es este logar, e la hora se passa; dexar ir las yentes a los castiellos a comprar que coman [Mateo, 46, 14:15]

b. otros dizen quele levara nuestro sennor Dios e que se fuera el pora El por la razón dantes desta salida de Egipto que fablara Dios alli con el [GEl, 467.24a]

En (12a) se observa que el verbo carece del clítico y la Meta es introducida por la preposición a. En cambio, en (12b) el verbo incluye en su construcción un clítico y una Meta introducida por la preposición para. La alta frecuencia de uso que las preposiciones a y para poseen en contextos similares, frente a otras menos frecuentes, y la escasa presencia bibliográfica que las oponen justifican el análisis realizado 5 . Es interesante establecer las motivaciones sintácticas o semánticas que llevaron al usuario del siglo XIII a alternar entre dos partículas directivas, como a y para. De lo anterior se formulan las siguientes preguntas: ¿̇exis-

4 Según el estudio de corpus Eslava (2013: 92) la preposición contra no rebasa $3 \%$; hasta, $1 \%$ y hacia, $1 \%$.

5 En general los estudios de preposiciones contrastan de la siguiente manera las preposiciones adlativas: Trujillo (1971) contrasta los significados de: a/hasta, para/por y para/hacia; Morera $(1998,1990)$ contrasta los significados de a/hasta, para/hacia y hacia/contra.

- Estos verbos están entre los prototípicos de movimiento incluidos en el bien conocido estudio tipológico de Talmy (1985) sobre patrones de lexicalización; en cuanto al español contemporáneo o actual, en particular, también estos verbos se reportan como de alta frecuencia, según el estudio de corpus de lbánéz Cerda $(2002: 170,2005)$ y, para el español alfonsí o siglo XIII, los resultados de análisis de corpus de Eslava Heredia (2013: 17) también los señala como de alta frecuencia. te dependencia entre el clítico y la selección de la preposición que introduce a la Meta? y ¿̇cuál sería entonces la función del clítico en las oraciones con verbos de movimiento intransitivos en el español del siglo XIII?

Lo que se propone aquí es que el clítico afecta al locativo Meta degradando su estatus argumental y tal degradación se formaliza con la selección de la preposición directiva para. La elección de esta preposición podría constituir un paso intermedio de la función de clítico como un «habilitador de categoría vacía» vía hacia el perfilamiento de la Fuente en oraciones con verbos de movimiento, tal como propone Ibáñez Cerda (2002), para etapas más tardías de la lengua española.

\section{MATERIALES Y MÉTODOS}

Para el análisis se recurrió a un corpus cerrado conformado por textos representativos del siglo XIII. El conjunto textual usado en el análisis procuró mostrar variedad temática: sapiencial, legal, crónica y religiosa. Con el fin de mantener un control cuantitativo, se utilizó un promedio de 21,000 palabras por género textual, lo cual sumó un total de 84,000 palabras. En este estudio particular se trabajó con tres verbos prototípicamente intransitivos y altamente frecuentes en el español del siglo XIII: ir, venir y tornarb.

Estos verbos manifiestan también una alta frecuencia de locativo explícito según lbáñez Cerda (2005: 192) y Eslava Heredia (2013: 55) y, principalmente, privilegian la expresión de un locativo Meta o destino. Se trata de verbos intransitivos, particularmente, del tipo inacusativo, ya que su sujeto es el que cambia de lugar o se ve afectado con dicho cambio (Mendikoetxea, 1999a: 1606-1607,1999b: 1700-1701). Además, estos verbos cubren todas las fases del movimiento $y$, por tanto, reproducen isomórficamente una estructura conceptual general de toda la clase; es decir, designan el movimiento como un desplazamiento completo de un punto de partida a uno de arribo (Ibáñez Cerda, 2002, 2005). De lo anterior, estos verbos se agrupan aspectualmente, como realizaciones o accomplishments, como los denomina Dowty (1979). Esta subclasificación de carácter aspectual permite, como lo propone Ibáñez Cerda (2005), derivar el estatus argumental de los complementos locativos que acompañan a los verbos en sus proyecciones oracionales (cf. Horno Chéliz, 1998), al menos de una manera general, sin que por ello se restrinjan del todo sus posibilidades construccionales. 
Por último, es conveniente el apoyo de algunas definiciones o características de las preposiciones directivas estudiadas aquí. Según Morera (1998: 239) las preposiciones a y para en los orígenes de la lengua podrían haber presentado los siguientes rasgos definitorios: a tenía un usentido adlativo finitivo sin extensión»; mientras que para mostraría un «sentido adlativo initivo con determinación al límite»». Así la preposición a implica la idea final del movimiento de aproximación a un límite, esto supone entonces mayor determinación; mientras que la forma para remite al inicio del movimiento de aproximación a un límite $y$, por tanto, se relaciona con una menor determinación de la meta. Recordemos también que, para usos actuales, Morera redefine los rasgos de para en función de los movimientos de significado que sufren las preposiciones hacia y contra, también initivas: a en la actualidad tendría un usentido adlativo finitivo sin extensión»; mientras que para mostraría un «sentido adlativo initivo con límite que se alcanza».

\section{RESULTADOS}

Como primer factor de análisis se determinó cuantitativamente la ocurrencia explícita de complementos de carácter locativo en las construcciones de los verbos analizados: ir, venir y tornar. En la Tabla 1 se observa que los porcentajes muestran que, para el siglo estudiado, existe una alta frecuencia de presencia del complemento locativo; al menos el total porcentual es de $67 \%^{7}$ de frecuencia relativa de uso de este adyacente verbal.

Un segundo factor de análisis altamente relevante en términos cuantitativos es la presencia 0 ausencia de un clítico pronominal. En la Tabla 2 es
Tabla 1. Presencia vs, ausencia de complemento locativo (CLOC)

\begin{tabular}{|c|c|c|}
\hline Verbo & $\begin{array}{c}\text { Presencia } \\
\text { cloc }\end{array}$ & $\begin{array}{c}\text { Ausencia } \\
\text { cLoc }\end{array}$ \\
\hline Ir & $68 \%(186 / 272)$ & $32 \%(86 / 272)$ \\
\hline Venir & $58 \%(139 / 238)$ & $42 \%(99 / 238)$ \\
\hline Tornar & $86 \%(78 / 91)$ & $14 \%(13 / 91)$ \\
\hline Total & $67 \%(403 / 601)$ & $33 \%(198 / 601)$ \\
\hline
\end{tabular}

posible observar con claridad tendencias muy interesantes en cuanto a las relaciones que se establecen entre el verbo en su forma pronominal y la presencia del complemento locativo.

Dos tendecias porcentuales llaman la atención en los datos de la Tabla 2: 1) se observan altos concentrados de copresencia del clítico y el complemento locativo Meta: $71 \%$ de las documentaciones con ir(se), $100 \%$ con venir(se), $81 \%$ con tornar(se), lo cual ya apunta a que no se trata de un clausurador de argumento per se; y 2) se ve un ligero declive en la presencia del locativo en casos sin el clítico: ir decae a $67 \%$ y venir, a $58 \%$. El verbo tornar, por su parte, en su lectura meramente intransitiva, mostró una tendencia contraria, pues cuando no hay clítico existe una mayor presencia del complemento locativo. Esto último tal vez se deba a que es el locativo el que permite distinguir entre ciertas lecturas transitivas e intransitivas, al menos en casos donde no media el clítico (p.ej. el mur tornó su cabeza vs. el mur tornó a su forado). Los totales al final de la Tabla 2 son claros: hay mayor copresencia del clítico y el locativo, $77 \%$; mientras que sin el clítico hay un descenso de presencia locativa: $64 \%$.

Tabla 2. Distribución de la presencia y ausencia de SE según aparezca o no el complemento locativo

\begin{tabular}{|c|c|c|c|c|}
\hline Verbo & \multicolumn{2}{|c|}{+ SE } & -SE \\
\hline & + CLOC & $-C L O C$ & $67 \%(110 / 165)$ & $33 \%(55 / 165)$ \\
\hline Ir & $71 \%(76 / 107)$ & $29 \%(31 / 107)$ & $58 \%(124 / 212)$ & $42 \%(88 / 212)$ \\
\hline Venir & $100 \%(16 / 16)$ & --- & $95 \%(20 / 21)$ & $5 \%(1 / 21)$ \\
\hline Tornar & $81 \%(42 / 52)$ & $19 \%(10 / 52)$ & $64 \%(254 / 398)$ & $36 \%(144 / 398)$ \\
\hline Total & $77 \%(134 / 175)$ & $23 \%(41 / 175)$ & $6 C$ & \\
\hline
\end{tabular}

7 Estos números coinciden con los resultados ofrecidos por lbánéz Cerda (2005: 192) para el español contemporáneo, pues reporta que el promedio porcental de presencia del complemento locativo en el grupo general de verbos de movimiento alcanza hasta $65 \%$. 
La ausencia del locativo Meta parece no implicar el desconocimiento del referente, debido a que este es inferido fácilmente o recuperable del contexto discursivo, véase los ejemplos de (13).

(13) a. E dixol Ihesus: Cata que lo non digas a ninguno; mas ve e muestra te al sacerdot, e offrez la offrenda que mando Moysen, en testimonio a ellos [Mateo, 33, 8:4]

b. Eł dixo él: -Non devieras tú dezir çerca del pozo, pues yo avía de ir al caño. Dixo ella: -¡Ve, et dexa la locura de ir et de venir! Dixo él: - ¿Cómmo iré, aviéndome tú conturbado? [Calila, 112]

c. E quando fue noche, uino un omne rico que auie nombre Joseph e era diciplo de lhesu Christo; aquel fue a Pilatus e pidio el cuerpo de Ihesu Christo [Mateo, 72, 27:57]

d. Yo vos digo verdat, que no acabaredes las ciudades de Israel fasta que uenga el Fi del ombre [Mateo, 37, 10:123]

En el caso de (13a) la Meta implícita es el Templo en Jerusalén y se infiere a través del conocimiento compartido entre los interlocutores sobre el lugar donde se debe acudir para llevar las ofrendas a Dios, según las leyes de Moisés; en (13b) los personajes discuten sobre la Meta que debe ser alcanzada, en este caso el caño. En (13c) la Meta es el escenario donde vino Joseph, el cual se clarifica en la oración posterior: "fue a Pilatos», en otras palabras, la Meta es el lugar de dominio de este gobernador. Por último, en (13d) la meta de venir se ubica en la oración anterior inmediata: las ciudades de Israel.

Volviendo a los datos, ahora se observa la correlación entre clítico y elección de preposiciones alternantes. En la Tabla 3 los datos muestran que la preposición a es la forma más empleada en las documentaciones para indicar la Meta, en oposición con el uso de para.

Los datos son contundentes: el uso del pronominal parece ser un condicionante para la selección de la preposición para, la cual alcanza $85 \%$ de los casos documentados; mientras que la frecuencia relativa de uso de la preposición a es de $21 \%$ cuando el verbo aparece con el clítico. Esta última, no obstante, no se encuentra del todo restringida en uso, pues ocurre en ambos contextos, con clítico y sin él, como en los ejemplos (14a) y (14b), repectivamente.
Tabla 3. Distribución promedio de preposiciones según la presencia o ausencia del clítico pronominal

\begin{tabular}{|c|c|c|}
\hline Preposición & A & PARA \\
\hline SE+ & $21 \%$ & $\begin{array}{c}85 \% \\
(45 / 214)\end{array}$ \\
\hline SE- & $79 \%$ & $15 \%$ \\
\hline TOTAL & $(169 / 214)$ & $(10 / 65)$ \\
\hline & $100 \%$ & $100 \%$ \\
& $(214 / 214)$ & $(65 / 65)$ \\
\hline
\end{tabular}

(14) a. E quando Ihesu Christo ovo acabadas estas palauras, fuesse de Galilea, e se uino a tierra de Iuda allend lordan, e sigueron les grandes compannas, e sano los alli [Mateo, 53, 19:1]

b. la carnesçaria que sea suya, \& el montaçenagdo; \& el abbadesa \& el convento que ayen la vintena de la caça que veniere al pueblo a vender [DLE, 388.286.34]

Por el contrario, la preposición para muestra mayor obstáculo en su elección y, por ende, su frecuencia es menor. Parece, entonces, existir una relación entre la presencia del clítico y la posibilidad de seleccionar entre la preposición a y la preposición para. La Tabla 3 manifiesta perfectamente las tendencias de uso de cada una de las preposiciones y podemos ver que los concentrados porcentuales se distribuyen de la siguiente forma: la preposición a ocurre de manera constante en $79 \%$ de las construcciones carentes del clítico; mientras que la preposición para aparece en $85 \%$ con el clítico. La Tabla 3 muestra claramente que hay una mayor posibilidad de uso de la preposición para en presencia del clítico que cuando no la hay. Esto es posible validarlo a través de la aplicación de la formula estadística de la $X^{2}$. Para ello, se plantearon dos hipótesis, una hipotesis nula $\left(\mathrm{H}_{\circ}\right)$ y una hipótesis alternativa $\left(\mathrm{H}_{\mathrm{A}}\right)$ que se describen a acontinuación:

$\mathrm{H}_{0}=$ No existe una relación entre el clítico y la elección de la preposición.

$\mathrm{H}_{\mathrm{A}}=$ Existe una relación entre el clítico y la elección de la preposición.

El rango de error es de 0.05 y el grado de libertad de 1. La Tabla 4 muestra los resultados de $X^{2}$ aplicada a los datos analizados.

$$
X^{2}=\sum \frac{\left(f_{o}-f_{e}\right)^{2}}{f_{e}} \quad \begin{aligned}
& X^{2} \text { tabular } 3.8415 \\
& X_{\text {calculada }}: 87.6848
\end{aligned}
$$


Tabla 4. Distribución $X^{2}$ de la relación clítico y preposiciones

\begin{tabular}{|c|c|c|c|c|c|c|}
\hline \multirow[t]{2}{*}{ Preposición } & \multicolumn{2}{|c|}{ Frecuencia observada } & \multicolumn{2}{|c|}{ Frecuencia teórica esperada } & \multirow[t]{2}{*}{ Total } & \\
\hline & $\mathrm{FO}_{\mathrm{A}}$ & $\mathrm{FO}_{\text {PARA }}$ & $\mathrm{FE}_{\mathrm{A}}$ & $\mathrm{FE}_{\text {PARA }}$ & & \\
\hline$S E+$ & 45 & 55 & 76.7025 & 23.2975 & 100 & \\
\hline SE- & 169 & 10 & 137.2975 & 41.7025 & 179 & Total $X^{2}$ \\
\hline Total & 214 & 65 & & & 279 & 87.6848 \\
\hline
\end{tabular}

$\mathrm{FO}=$ frecuencia observada, $\mathrm{FE}=$ frecuencia teórica esperada, $\mathrm{GL}=1, \alpha 0.05$.

La aplicación de la formula estadística de la $X^{2}$ a los datos de esta investigación permite rechazar la $\mathrm{H}_{\mathrm{o}}=$ No hay una relación entre clítico y la elección de preposición y aceptar la $\mathrm{H}_{\mathrm{A}}=$ Existe una relación entre el clítico y la elección de la preposición con una significación del 0.05 de margen de error, ya que al comparar la $X^{2}$ tabular (3.8415) con la $X^{2}$ calculada (87.6848), esta última es mayor que la primera; lo que permite descartar la hipótesis nula. Ahora obsérvense los datos arrojados por cada uno de los verbos analizados en las Tablas 5 a 7 . Cada tabla muestra la distribución de las preposiciones, según la presencia o ausencia del clítico pronominal, en cada uno de los verbos seleccionados para este trabajo.

Tabla 5. Distribución de preposiciones con o sin el clítico en el verbo ir

\begin{tabular}{|c|c|c|}
\hline \multirow{2}{*}{} & \multicolumn{2}{|c|}{ IR } \\
\cline { 2 - 3 } & $\boldsymbol{A}$ & PARA \\
\hline \multirow{2}{*}{ SE+ } & $27 \%$ & $88 \%$ \\
& $(25 / 94)$ & $(36 / 88)$ \\
\hline \multirow{2}{*}{ SE- } & $73 \%$ & $12 \%$ \\
& $(69 / 94)$ & $(5 / 12)$ \\
\hline \multirow{2}{*}{ TOTAL } & $70 \%$ & $30 \%$ \\
& $(94 / 135)$ & $(41 / 135)$ \\
\hline
\end{tabular}

Tabla 6. Distribución de preposiciones con o sin el clítico en el verbo venir

\begin{tabular}{|c|c|c|}
\hline \multirow{2}{*}{} & \multicolumn{2}{|c|}{ VENIR } \\
\cline { 2 - 3 } & $\boldsymbol{A}$ & PARA \\
\hline \multirow{2}{*}{$S E+$} & $5 \%$ & $90 \%$ \\
& $(4 / 78)$ & $(9 / 10)$ \\
\hline SE- & $95 \%$ & $10 \%$ \\
& $(74 / 78)$ & $(1 / 10)$ \\
\hline TOTAL & $89 \%$ & $11 \%$ \\
& $(78 / 88)$ & $(10 / 88)$ \\
\hline
\end{tabular}

Tabla 7. Distribución de preposiciones con o sin el clítico en el verbo tornar

\begin{tabular}{|c|c|c|}
\hline \multirow{2}{*}{} & \multicolumn{2}{|c|}{ TORNAR } \\
\cline { 2 - 3 } & $\boldsymbol{A}$ & PARA \\
\hline \multirow{2}{*}{ SE+ } & $38 \%$ & $71 \%$ \\
& $(16 / 42)$ & $(10 / 14)$ \\
\hline \multirow{2}{*}{ SE- } & $61 \%$ & $29 \%$ \\
& $(26 / 42)$ & $(4 / 14)$ \\
\hline \multirow{2}{*}{ TOTAL } & $75 \%$ & $25 \%$ \\
\hline
\end{tabular}

Las Tablas 5, 6 y 7 vuelven a manifestar claramente, por un lado, que la preposición a ocurre generalmente cuando está ausente del clítico: $73 \%$ con el verbo ir, $95 \%$ con el verbo venir y tornar con un $61 \%$, como en los ejemplos de (15a-c).

(15) a. E† yo iré a la cueva, et entraré en ella, et si fallare la serpente muerta, tomaré su çerebro et aduzírtelo he [Calila, 340]

b. Ell anno en que Moysen salio de Egipto desta vez e vino a Madian, avie el quarenta e dos annos que nasciera [GEI, 321.2a]

c. [un espíritu inmundo] Estonce dize: Tornare a mi casa dond Sali [Mateo, 42, 12:44]

Por otra parte, la preposición para aparece en mayor porcentaje en todos los verbos cuando las construcciones presentan el clítico se: $88 \%$ con el verbo irse, $90 \%$ con el verbo venirse y $71 \%$ con el verbo tornarse, tal como se ejemplifica en (16a-c).

(16) a. e maguer que les dixiera quando se yua poral mont que XLa días e XLa noches durarie alla, non pararon ellos mientes en la cuenta de tantos días nin de tantas noches, [GEl, 468.46a] b. Dizen que una culebra envegeçió et enflaqueçió, et non podía caçar; et vínose para una fuente do avía muchas ranas de que ella solía caçar [Calila, 248] 
c. E después que esto ovo fecho, dixo que se quería tornar para su tierra e posó en casa de un omne bueno [Sendebar, 132]

De igual manera, se aplicó la fórmula de $X^{2} a$ los datos de cada verbo por separado, siguiendo igualmente un margen de error del 0.05, con un grado de libertad de 1, para las dos hipótesis antes formuladas:

$\mathrm{H}_{0}=$ No existe una relación entre el clítico y la elección de la preposición y

$\mathrm{H}_{\mathrm{A}}=$ Existe una relación entre el clítico y la elección de la preposición.

Los resultados fueron los siguientes:

Tabla 8. Distribución $X^{2}$ del clítico y la preposición en el verbo ir

\begin{tabular}{|c|c|c|c|c|c|c|}
\hline IR & \multicolumn{2}{|c|}{ Frecuencia observada } & \multicolumn{2}{|c|}{ Frecuencia teórica esperada } & Total & \\
\hline & $\mathrm{FO}_{\mathrm{A}}$ & $\mathrm{FO}_{\text {PARA }}$ & $\mathrm{FE}_{\mathrm{A}}$ & $\mathrm{FE}_{\text {PARA }}$ & & \\
\hline $\mathrm{SE}+$ & 25 & 36 & 42.4741 & 18.5259 & 61 & \\
\hline $\mathrm{SE}-$ & 69 & 5 & 51.52 .59 & 22.4741 & 74 & Total $^{2}$ \\
\hline Total & 94 & 41 & & & 135 & 43.1834 \\
\hline
\end{tabular}

GL 1, $\propto 0.05, X^{2}$ tabular $3.8415, X^{2}$ calculada 43.1834.

Tabla 9. Distribución $X^{2}$ del clítico y la preposición en el verbo venir

\begin{tabular}{|c|c|c|c|c|c|c|}
\hline VENIR & \multicolumn{2}{|l|}{ Frecuencia observada } & \multicolumn{2}{l|}{ Frecuencia teórica esperada } & Total & \\
\hline & $\mathrm{FO}_{\mathrm{A}}$ & $\mathrm{FO}_{\mathrm{PARA}}$ & $\mathrm{FE}_{\mathrm{A}}$ & $\mathrm{FE}_{\mathrm{PARA}}$ & & \\
\hline $\mathrm{SE}+$ & 4 & 9 & 11.5227 & 1.4773 & 13 & \\
\hline SE- & 74 & 1 & 66.4773 & 8.5227 & 75 & Total $X^{2}$ \\
\hline Total & 78 & 10 & & & 88 & 50.7096 \\
\hline
\end{tabular}

GL 1, $\propto 0.05, X^{2}$ tabular $3.8415, X^{2}$ calculada 50.7096 .

Tabla 10. Distribución $X^{2}$ del clítico y la preposición en el verbo tornar

\begin{tabular}{|c|c|c|c|c|c|c|}
\hline TORNAR & \multicolumn{2}{|c|}{ Frecuencia observada } & \multicolumn{2}{|c|}{ Frecuencia teórica esperada } & Total & \\
\hline & $\mathrm{FO}_{\mathrm{A}}$ & $\mathrm{FO}_{\text {PARA }}$ & $\mathrm{FE}_{\mathrm{A}}$ & $\mathrm{FE}_{\text {PARA }}$ & & \\
\hline $\mathrm{SE}+$ & 16 & 10 & 19.5000 & 6.5000 & 26 & \\
\hline $\mathrm{SE}-$ & 26 & 4 & 22.500 & 7.5000 & 30 & Total $X^{2}$ \\
\hline Total & 42 & 14 & & & 56 & 4.6906 \\
\hline
\end{tabular}

GL $1, \alpha 0.05, X^{2}$ tabular $3.8415, X^{2}$ calculada 4.6906 .

Como se puede observar en cada una de las tablas, las $X^{2}$ calculadas son mayores a la $X^{2}$ tabular, lo cual permite, una vez más, rechazar la $\mathrm{H}_{0}$ y aceptar la $\mathrm{H}_{\mathrm{A}}$, con un margen de error de 0.05 .

\section{DISCUSIÓN}

Como se aprecia en las Tablas 3, 5, 6, 7 y la distribución de $X^{2}$ en las Tablas 4, 8, 9 y 10, el elemento motivador para la alternancia entre a y para es claramente la presencia del clítico, pero żqué relación se establece entre el clítico y la preposición para? 
Según se observa, la presencia del clítico abre la opción de uso de la preposición para; se dice opción, porque el uso de la preposición a se mantiene en ambos contextos formales (hay que recordar los resultados en cada tabla: $27 \%$ con ir; $5 \%$ con venir y $38 \%$ con tornar). No obstante, el uso de la preposición para parece sugerir que el clítico ha debilitado o degradado el carácter argumental o valencial del complemento locativo Meta, pues es posible lograr lecturas comprensibles de las mismas oraciones, aun cuando estas carezcan de complemento locativo. Contrástese las oraciones de (17) con las construcciones de (18). Obsérvese que la supresión de la Meta introducida con la preposición para no conlleva a la agramaticalidad de las oraciones.

(17) E† pues que se fue la madre del león para su casa, et pasó la media noche, dixeron a Calila cómmo Digna era preso [Calila, 188]

E desque esto ovieron hecho, tornáronse para la hueste; e entraron de noche bien aquella ora que della partieran [Conquista, 635]

(18) E† pues que se fue la madre del león [...], et pasó la media noche, dixeron a Calila cómmo Digna era preso

E desque esto ovieron hecho, tornáronse [...]; e entraron de noche bien aquella ora que della partieran

El reflejo de la degradación del carácter argumental se formaliza con la elección de una preposición para, cuyas características impactan en un doble frente. Por un lado, el rasgo "initivo" de la preposición remite al inicio del movimiento, lo que implica mayor indeterminación de la Meta; por consecuencia, menor grado de argumentabilidad y mayor facilidad de supresión como se observa en (18), por ello se justifica la función del clítico como "habilitador de categoría vacía". Por otro lado, cuando coaparecen el clítico y la preposición para, el "sentido de límite que se alcanza" de la última comulga bien con el sentido de completud o término que añade el clítico al verbo que propone Bogard Sierra (2006).

Evidentemente, el clítico podría funcionar como clausurador de argumento en ciertos verbos, como meter o internarse, que requieren este pronombre para su lectura intransitiva (Juan metió el perro a la casa/Juan se metió a la casa), donde el argumento clausurado es un objeto directo, argumento interno del verbo. No obstante, el juego de preposiciones en los verbos analizados aquí sugiere que el clítico funciona, en primer lugar, como un "habilitador de categoría vacía" debido a que degrada el estatus argumental de la Meta, pero sin llegar a ser un "clausurador argumental", dada la persistencia de la Meta; en segundo lugar, como una "marca aspectual", como lo describe Bogard Sierra (2000, 2006: 768-769); esto es, el clítico también perfila una lectura de completud o término de la acción. Lo anterior explicaría, en esta etapa temprana del español, la constante ocurrencia del locativo Meta en presencia del clítico.

\section{CONCLUSIONES}

Se ha observado que la función del clítico y la formalización del locativo Meta tienen que ver con la compatibilidad de rasgos que cada elemento aporta a la oración. Recordemos que la preposición a tiene un sentido "finitivo»; es decir, pone en foco la fase final del movimiento, esto supone una mayor determinación de la Meta y su prefiguración como un elemento argumental; mientras que para desde su origen pone en foco la fase inicial del movimiento: la idea de aproximación se vuelve más indeterminada; por tanto, la Meta se interpreta como menos argumental. De ahí que la función del clítico como «habilitador de categoría vacía» se formalice en la selección de la preposición para. Por otra parte, el sentido de propósito definido o intencional de alcanzar el límite de para es afín a la idea de perfectividad que añade el clítico a la acción. De este modo, la elección de las preposiciones a/para constituye un paso gradual del clítico con función de «habilitador de cate-

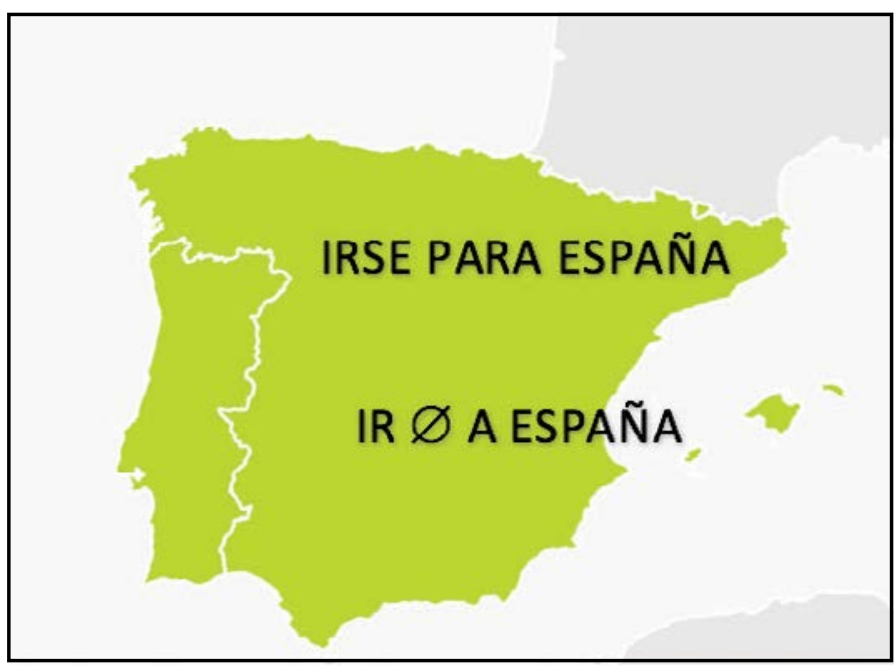

Figura 1. Mapa de España modificado con base en el publicado en el portal electrónico TomTom International BV (2016). 
goría vacía», concomitante con la función de «marca aspectualı, que va hacia la función de cambio de perspectiva de la Fuente sobre la Meta en este tipo de verbos de movimiento. El trabajo aquí presentado brinda información importante que apoya la hipótesis del clítico se como «habilitador de categoría vacía» y «marcador aspectual») (según propone Bogard Sierra, 2006) y contribuye a la mejor comprensión del uso de preposiciones y las pautas de comportamiento que las rigen en el periodo de estudio.

\section{LITERATURA CITADA}

- bOGARD SIERRA, S. El clítico reflexivo como marcador aspectual en español. Tópicos del Seminario, 3: Aspectualidad y modalidades, 41-48, 2000.

- BOGARD SIERRA, S. El clítico se. Valores y evolución. En C. Company Company (Ed.), Sintaxis histórica de la lengua española. Primer parte: La frase verbal. Volumen 2 (pp. 755-870). México: Fondo de Cultura Económica-Universidad Nacional Autónoma de México, 2006.

- CREgO GARCíA, M. V. El complemento locativo en español, los verbos de movimiento y su combinatoria sintáctico-semántica. Colección LALIA, series MAIOR, no. 12. Santiago de Compostela, España: Servicio de Publicacións e Intercambio Cientifico, 2000.

- DOWTY, D. R. Word meaning and montague grammar. Dordrecht: Reidel, 1979.

- ESLAVA HEREDIA, C. Verbos de movimiento en el español alfonsí. Análisis sintáctico-semántico. Aguascalientes, México: Universidad Autónoma de Aguascalientes, 2013.

- FILLMORE, C. J. Frames Semantics. En THE LINGUISTIC SOCIETY OF KOREA (Ed.), Linguistics in the morning calm. Selected papers from SICOL-1981 (pp. 113-137). Seoul, Korea: Hanshing Publishing Company, 1982

- GarCía MIGUEL, J. M. Los complementos locativos. En C. Company Company (Ed.), Sintaxis histórica de la lengua española. Primer parte: La frase verbal. Volumen 2 (pp. 12531333). México: Fondo de Cultura Económica-Universidad Nacional Autónoma de México, 2006.

- HORNO CHÉLIZ, M. C. Conceptualización y categorización lingüística de las relaciones espaciales en verbos locativos. En J. L. CIFUENTES HONRUBIA (Ed.), Estudios de lingüística cognitiva II (pp. 629-637). España: Universidad de Alicante, 1998.

- IBÁÑ̃EZ CERDA, S. El clítico SE en los verbos de movimiento intransitivos del español. Un análisis en términos de estructura temática. Nueva Revista de Filología Hispánica, L(1): 169-180, 2002.

- IBÁÑEZ CERDA, S. Los verbos de movimiento intransitivos del español. Una aproximación léxico-sintáctica. México: INAHUNAM, 2005.

- MENDIKOETXEA, A. Construcciones inacusativas y pasivas. En I. BOSQUES y V. DEMONTE (Dirs.), Gramática descriptiva de la Lengua Española 2. Las construcciones sintácticas fundamentales. Relaciones temporales, aspectuales y modales (pp. 1575-1629). Madrid: Espasa Calpe, 1999a.

- MENDIKOETXEA, A. Las construcciones con se: medias, pasivas e impersonales. En I. BOSQUES y V. DEMONTE (Dirs.), Gramática descriptiva de la Lengua Española 2. Las construcciones sintácticas fundamentales. Relaciones temporales, aspectuales y modales (pp. 1631-1722). Madrid: Espasa Calpe, 1999b.

- MORERA, M. La preposición española contra: su evolución semántica. Verba, 17, 287-313, 1990.

- MORERA, M. Origen y evolución de la preposición española hacia. Revista de Filología de la Universidad de La Laguna, 16, 231-243, 1998.

- TALMY, L. Semantics and syntax of motion. En J.P. KIMBALL (Ed.), Syntax and Semantics, 4 (pp. 181-238). Indiana University, Bloomington, Illinois, US: Academic Press, 1975.

- TALMY, L. Lexicalization patterns: semantic structure in lexical forms. En T. SHOPEN (Ed.), Lenguage tipology and syntactic description, 3: Grammatical categories and the lexicon (pp. 57149). Cambridge, England: Cambridge University Press, 1985

- tRUJILlO, R. Notas para un estudio de las preposiciones españolas. THESAURUS, Boletín del Instituto Caro y Cuervo, Tomo XXVI, Bógota, 1971.

\section{CORPUS}

- $[$ Calila $]=$ CACHO BLECUA J. M. / Ma. de J. LACARRA (Eds.), Calila e Dimna. Madrid: Castalia, 1998.

- [Conquista] COOPER, L. (Ed.), La gran conquista de Ultramar. Bógota: Publicaciones del Instituto Caro y Cuervo, LI, Tomo I, 1979.

- $\quad[D L E]=$ MENÉNDEZ PIDAL, R. (Ed.), Documentos lingüísticos de España I. Reino de Castilla. Madrid: Consejo Superior de Investigaciones Científicas, 1966.

- $[G E l]=S O L A L I N D E, A$. G. (Ed.), Alfonso X El Sabio. General Estoria. Primera parte. Madrid: Centro de Estudios Históricos, MCMXXX, 1930.

- $\quad[$ Mateo $]=$ MONTGOMERY, M. (Ed.), El Evangelio según San Mateo. Anejos del Boletín de la Real Academia Española. Madrid: REA. Anejo VII, 1962.

- [Sendebar] LACARRA, M. J. (Ed.), Sendebar. Madrid: Cátedra, 1989.

\section{De páginas electrónicas}

- TOM TOM INTERNATIONAL BV. Mapa de la península ibérica (España y Portugal) [Mapa]. Autor, 2016. Recuperado de https:// www.tomtom.com/es_es/drive/maps-services/shop/travelmap/iberia/\#\#) 